\title{
M-health for maternal health- bridging the gaps!!
}

\author{
Sheeba Marwah ${ }^{1 *}$, Pratima Mittal ${ }^{2}$ \\ ${ }^{1}$ Department of Obstetrics and Gynecology, NDMC Medical College and Hindu Rao Hospital, New Delhi, India \\ ${ }^{2}$ Department of Obstetrics and Gynecology, VMMC and Safdarjung Hospital, New Delhi, India
}

Received: 28 November 2017

Accepted: 18 December 2017

*Correspondence:

Dr. Sheeba Marwah,

E-mail: sheebamarwah@yahoo.co.in

Copyright: (C) the author(s), publisher and licensee Medip Academy. This is an open-access article distributed under the terms of the Creative Commons Attribution Non-Commercial License, which permits unrestricted non-commercial use, distribution, and reproduction in any medium, provided the original work is properly cited.

\begin{abstract}
This article reviews significance, potential and principles to consider when setting up a telemedicine (TM) program to provide care to women in the field of obstetrics and gynecology, essentially deploying mobile technology. There are various benefits of such TM clinical applications. The consensus among patients and health care providers is that this technology is convenient to provide needed subspecialty medical care, even when it is not available locally. Such innovations are clinically successful, but economic and cost-effectiveness data are lacking.
\end{abstract}

Keywords: Gynecology, Mobile health, Obstetrics, Telemedicine, Telehealth

\section{INTRODUCTION}

Every year, approximately 350,000 women die worldwide as a direct result of pregnancy and childbirth, and for each woman who dies, approximately 20 others suffer from pregnancy related consequences. ${ }^{1}$ Additionally, three million babies die every year before they are a month old, and a similar number are stillborn. ${ }^{2,3}$ When stillbirths are included, about half of all 7.5 million deaths in children under age five occur before the end of the neonatal period, or the first 28 days of life, and without a reduction in the number of neonatal deaths, a dent cannot be made in under-five mortality rates. ${ }^{4}$

Despite the availability of many evidence-based interventions for improving maternal health, ineffective implementation often leaves women lacking the care they need. ${ }^{5}$ Inadequate methods of reaching women with critical health information, lack of support for healthcare providers, and inconsistent use of data are just some of the numerable factors that constrain a health system's ability to deliver effective maternal healthcare. This makes it imperative to design effectual and innovative strategies to bridge the gaps.

\section{TELEHEALTH/TELEMEDICINE}

Innovation and implementation of tele-medicine (TM)/telehealth $(\mathrm{TH})$ programs are moving at a fast pace. TH is any health-related service that exploits electronic information and communication technology to dissipate health or medical information to a patient in a remote or mobile setting. TM is the usage of electronic information and communication technology for imparting direct patient health services when the health care medical provider and patient are separated by some physical distance. ${ }^{6,7}$ The premise of TM is that it will be of similar quality to in-person visits but more convenient and efficient for both the patient and the medical provider.

\section{Formats of $\mathrm{TM} / \mathrm{TH}$}

Possible formats in TM TH include "store and forward" models, videoconferencing, messaging or e-mail 
encounters, tele-phone calls, web sites, or smartphone applications. Remote monitoring of patient-level data such as vital signs, fetal heart rate monitoring, or blood sugars, can be sent electronically to a provider in a different location. Online and mobile health can provide health care information through the internet or to smart phones. TH encounters have been implemented in many medical specialties in western countries. ${ }^{6,7}$

\section{What is $m$ heath?}

m-Health (mobile health) is a general term for the use of mobile phones and other wireless technology in medical care, from the large gamut of TM and TH. The most common application of $\mathrm{m}$ Health is the use of mobile phones and communication devices to educate consumers about preventive health care services. It has now emerged as a strategic approach or tool when designing maternal health research, programs and service delivery. ${ }^{8}$

Health Information Systems (HIS) gather, aggregate, analyse and synthesize data from multiple sources to report on health; can include information related to patient records, disease surveillance, human resources, management of commodities, financial management, service delivery and other data needed for reporting and planning purposes.

\section{Rationale of $m$ health- gaps identified}

Barriers associated to the 3 delays leading to most pregnancy-related mortality to health care prevent women to benefit from life-saving interventions. These are delayed decision to seek medical care, delay in getting to the health center, delay in receiving adequate care when a facility is reached, and lack of proper Post-partum follow-up. Identifying these common yet important hurdles in road to health and safe motherhood would go a long way in improving health care imparted in the country.

Providers and health care systems may benefit from $\mathrm{m}$ health TM programs due to less travel to outreach clinics, provide care conveniently from their office, potentially lower costs for clinic infrastructure. Anganwadi workers and health workers at sub Centre levels may benefit from such TM programs as it keeps their patients local, gives better access to subspecialty providers and may keep other downstream revenues in the local health care system. ${ }^{9}$

\section{Key operational strategies of maternal health include}

- Access to a continuum of care, including appropriate management of pregnancy,

- Enable access to life-saving emergency obstetric care (EmONC) when complications arise

- Improving referral system

- Delivery in health facility by skilled birth attendant

- Monitoring during post-partum period

\section{Research and conceptualization of $m$ health}

Keeping all this in mind, many researchers across the globe have dug into the subject deeply and advocated the utilization of $\mathrm{m}$ Health to improve the effective delivery of maternal health services. ${ }^{4-13}$ They have concluded that mobile technology can be used to enhance the delivery of maternal health services by improving a client's knowledge and self-efficacy, a provider's competence, accountability and effectiveness; and, a health system's ability to manage its commodities, equipment and health workers.

Evidence for $\mathrm{m}$ Health Alliance and $\mathrm{MNCH}$ substantiates that Increase in rigorous $m$ Health research has been remarkable. It also calls for greater investment of resources in studying the effect $m$ Health interventions have on health outcomes. It emphasizes need to view gaps in the $\mathrm{m}$ Health evidence as opportunities for future research.

\section{Aims of m- health}

- To contribute to the reduction of delays associated with transport to the referral facility; associated with timely intervention when the mother reaches the referral facility.

- To provide real-time information regarding maternal health in India, especially at grass-root level through routine SMS-based data collection methods.

- To increase the number of women who attend the WHO recommended four antenatal care (ANC) visits and the number of facility deliveries through SMS reminders

\section{Rationale for using mobile phones}

The number of mobile phone subscribers in India rose to over 50 million following market liberalization in twenty first century. Besides. There has been a continued growth in coverage of mobile networks. Mobile phone penetration rate in the country is expected to reach $75 \%$ by end of 2020. Utilizing this unexplored potential of Mobile technology can be therefore used to enhance the delivery of maternal health services even at the grass-root levels.

\section{Why m health?}

Mobile phones have the potential to reduce delays that often result in maternal death: Delayed decision-making added to delayed transport. They are an important source to mitigate information-sharing problems across Health centers, facilities and Hospitals in the region. It can aid in expanding transportation and referral for maternal health. Mobile phones can promote continuity of care: follow-up at community level and information sharing for effective referral. They could also aid in point-of-care decisionmaking by the index physician. Also, it can update appointment reminders for the women enabling better 
antenatal care. They can also be a vital part of supply chain management, and enable disease tracking.

\section{Functioning- technical aspects}

A mobile phone SMS-based system Using Rapid SMS open-sourced platform will be employed designed and developed by $m$ Health team at MOHFW. This will ensure dynamic data collection and sharing.

\section{Five programmatic areas}

- Behavior Change Communication (BCC)

- Data collection

- Finance

- Logistics

- $\quad$ Service delivery

Innovations proposed under $m$ health as heath system strengthening tools

- Client education and behavior change communication

- Sensors and point-of-care diagnostics

- Registries and vital events tracking

- Data collection and reporting

- Electronic health records

- Electronic decision support: information, protocols, algorithms, checklists

- Provider-to-Provider Communication: User Groups, Consultation

- Provider work planning and scheduling

- Provider training and education

- Human resource management

- Supply chain management

- Financial transactions and incentives. ${ }^{14}$

\section{Pregnancy registration and follow up workflow}

Mothers' details are recorded into safe motherhood register, pregnancy is registered in SMS system. Through the server, the birth attendant or aanganwadi worker is notified of a new mother being registered in her zone of operation. She then keeps a record of the pregnant woman in a logbook and enables the patient to follow up in antenatal period through regular SMS alerts. Reports also exchanged and delivered through the same. M Mitra service is deployed in Safdarjung hospital based on this as a novel strategy. ${ }^{15}$ Such Virtual prenatal visits for lowrisk pregnant women do not replace routine in-person prenatal visits, but can be integrated into traditional prenatal care model. ${ }^{16}$

\section{Advantages of $m$ health}

Apart from evading the delays in seeking health care by women, m- health can keep less critical patients local and reduce the need for office visits, hospitalizations, and emergency department visits for non-emergent indications. ${ }^{17,18}$

\section{Challenges anticipated}

- Non-availability of baseline data necessary measure change over time

- $\quad$ Limited mobile network coverage in certain areas

- Illiteracy

- Financial constraints

- Lack of awareness

\section{Key factors for $m$ health success}

Potential for $m$ Health interventions is GREAT. Roll out of m-Health interventions requires Time and significant investment in human, technical and physical infrastructure, continuous capacity strengthening at multiple levels in the use of mobile technology devices, information dissemination, data collection, monitoring and evaluation, country Ownership and Leadership, Integration of m-Health into existing national and local health sector plans, strategies and systems, development and implementation of $\mathrm{m}$-Health-friendly policies, strong public-private partnerships and Coordination of efforts of all partners through establishment of a technical working group consisting of partner representatives.

\section{Way forward}

A thorough facility and community assessment is required to build $m$ Health system that meet the needs of intended beneficiaries. Efforts to catalogue and grade the $\mathrm{m}$-Health for $\mathrm{RMNCH}$ evidence base are underway (WHO mTERG). ${ }^{14}$ Involvement of trained Safe Motherhood Action Groups (SMAGs) or Traditional Birth Attendants (TBAs) in community based interventions/activities is contemplated. A bigger impact on maternal health will be achieve if communication and transport is improved at community level

\section{FUTURE}

Development of web user interface and System enhancement is the next step. Undertaking community based assessment of communication and transportation system, Documenting lessons learned and Generating evidences and evaluating the impact of the SMS system would be the future goals.

\section{CONCLUSION}

TM continues to evolve as a modality to provide care for patients in models that are drastically different from the traditional in-person encounter between physician and patient. M Health applied to maternal health has the potential to enable monitoring, follow-up and referrals of pregnant women along a continuum of care from the start of the pregnancy all the way through her post-partum period. Also, the use of mobile phones will reduce 
delayed decision-making and transportation during childbirth. However, specific to applications of TM and m-health in OBGYN, there are questions of whether this trade-off compromises medical care, are health outcomes similar or improved when compared with in-person visits and is it a cost-effective way to provide/ supplement care to women in a resource poor setting like India. Further research in this field to answer these questions, when $\mathrm{TM}$, especially $\mathrm{m}$ health will be universally integrated in nation's health model.

Funding: No funding sources Conflict of interest: None declared

Ethical approval: Not required

\section{REFERENCES}

1. Public Health. Focus on mHealth: WHO mHealth Technical Advisory Group for evidence in reproductive, maternal, newborn and child health. K4 Health Newsletter Special Supplement. 2013;1(2).

2. Fraser H, Bailey C, Sinha C, Mehl G, Labrique AB. Call to action on global eHealth evaluation: consensus statement of the WHO Global eHealth Evaluation Meeting, Bellagio;September 2011.

3. World Health Organization (WHO). The world health report 2000 - health systems: improving performance. Geneva: WHO; 2000. Available at: http://www.who.int/whr/2000/en/

4. Travis P, Bennett S, Haines A, Pang T, Butta Z, Hyder AA et al. Overcoming health-systems constraints to achieve the Millennium Development Goals. Lancet. 2004; 364(9437):900-6.

5. Center for Innovation and Technology in Public Health, Public Health Institute; mHealth Alliance. Leveraging mobile technologies to promote maternal and newborn health: the current landscape and opportunities for advancements in low-resource settings. Washington, DC: mHealth Alliance; [2012]. Available http://www.healthunbound.org/sites/default/files/ uploads/leveraging_mobile_technologies_to_promot e_maternal_newborn_health.pdf

6. Field MJ. Telemedicine: a guide to assessing telecommunications for health care. Washing-ton, DC: National Academy Press;1996.

7. Greiner AL. Telemedicine Applications in Obstetrics and Gynecology. Clin Obstet Gynecol. 2017;60(4):853-66.
8. Bashshur R, Shannon G, Krupinski E, Grigsby J. The taxonomy of telemedicine. Telemed J E Health. 2011;17(6):484-94.

9. Asch DA. The hidden economics of telemedicine. Ann Intern Med. 2015;163:801-2.

10. Earth Institute, Columbia University. Barriers and gaps affecting mHealth in low and middle-income countries: a policy white paper. Washington, DC: mHealth Alliance; 2010. Available at http://cghed.ei.columbia.edu/sitefiles/file/ m Health Barriers White PaperFINAL.pdf

11. MCTS (Mother and Child Tracking System) [Internet]. India: Ministry of Health and Family Welfare, Department of Health and Family Welfare, National Rural Health Mission (NRHM); [cited 2013 Mar 13]. Available at http://nrhm-mcts.nic.in/MCH/2

12. Tamrat $\mathrm{T}$, Kachnowski S. Special delivery: an analysis of mHealth in maternal and newborn health programs and their outcomes around the world. Matern Child Health J. 2012;16(5):1092-1101.

13. MAMA: Mobile Alliance for Maternal Action [Internet].Washington, DC: United Nations Foundation; c2013 [cited 2013 Mar 13]. Available at: http://mobilemamaalliance.org/

14. Labrique AB, Vasudevan L, Kochi E, Fabricant R, Mehl G. mHealth innovations as health system strengthening tools: 12 common applications and a visual framework. Global Health: Sci Pract. 2013;1(2):160-71

15. Derenzi B, Borriello G, Jackson J, Kumar VS, Parikh TS, Virk P, et al. Mobile phone tools for field-based health care workers in low-income countries. Mt Sinai J Med. 2011;78(3):406-18.

16. Butler Tobah YS. OB nest: a novel approach to prenatal care. Obstet Gynecol. 2016;127:7S-8S.

17. Beatty Y. Current telemedicine landscape in Tennessee. Tennessee Med. 2015;108:23-27.

18. Okoroh EM, Kroelinger CD, Smith AM, Goodman DA, Barfield WD. US and territory telemedicine policies: identifying gaps in perinatal care. Am J Obstet Gynecol. 2016;215:e1-772.e6.

Cite this article as: Marwah S, Mittal P. M-health for maternal health- bridging the gaps!!. Int J Reprod Contracept Obstet Gynecol 2018;7:1-4. 